\title{
Villes-usines et company towns en Europe de l'ouest et en Amérique du Nord : caractéristiques, synthèse, potentiel postindustriel
}

Villes-usines and company towns in West Europe and North America : patterns, overview, postindustrial potential

\section{Michaël Picon}

\section{(2) OpenEdition}

\section{Journals}

Édition électronique

URL : http://journals.openedition.org/rge/9316

DOI : $10.4000 /$ rge. 9316

ISSN : 2108-6478

Éditeur

Association des géographes de l'Est

Référence électronique

Michaël Picon, « Villes-usines et company towns en Europe de l'ouest et en Amérique du Nord:

caractéristiques, synthèse, potentiel postindustriel », Revue Géographique de l'Est [En ligne], vol. 58/3-4

2018, mis en ligne le 23 juillet 2020, consulté le 08 septembre 2020. URL : http://

journals.openedition.org/rge/9316 ; DOI : https://doi.org/10.4000/rge.9316

Ce document a été généré automatiquement le 8 septembre 2020.

Tous droits réservés 


\section{Villes-usines et company towns en Europe de l'ouest et en Amérique du Nord : caractéristiques, synthèse, potentiel postindustriel}

Villes-usines and company towns in West Europe and North America : patterns, overview, postindustrial potential

Michaël Picon

\section{Introduction}

«Les cités industrielles se pressent dans le voisinage des puits de mines ; la population s'y masse en foules épaisses sur un sol noirci par les débris de charbon, sous un ciel fuligineux où l'on cherche vainement à discerner le soleil. Mais l'étude plus approfondie des forces de la nature suscite de nos jours de nouveaux serviteurs à l'industrie humaine; l'eau qui se précipite du haut des montagnes est, comme la houille, productrice d'énergie et se transforme en mouvements innombrables pour façonner la matière. L'industriel commence à se déplacer ; des cités nouvelles surgissent dans les vallées, des monts au milieu des pâturages et des forêts ; les rudes ouvriers succèdent aux pâtres et aux bûcherons. » (Reclus 1905, p. 328 et 329).

Cette citation très naturaliste du géographe Élysée Reclus décrit de manière quasi universelle la genèse des villes-usines occidentales du XIXe siècle. Celles-ci sont nées de la volonté patronale de sortir des faubourgs industriels pour investir des espaces ruraux, plus sains (sous l'influence de l'hygiénisme ou labor environmentalism), d'un milieu favorable à l'industrie (ressources naturelles), d'avancées techniques et, souvent, d'autres éléments de contexte particuliers (passé proto-industriel, enjeux géopolitiques, etc.). Ces villes-usines sont pensées, organisées et aménagées par des patrons paternalistes: elles sont la réalisation spatiale du paternalisme industriel: "Paternalisme et philanthropie se sont exprimés dans l'espace et dans le bâti 》 (G. Dorel-Ferré 2016, p. 10). L'espace y est marqué par l'hégémonie des patrons paternalistes 
(omniprésence symbolique, contrôle social et politique, cocon paternaliste, etc.) et l'organisation spatiale du travail dans la ville. Les cousines nord-américaines des villesusines sont fortement influencées par les idées et les réalisations européennes, nous verrons qu'il en existe une variété assez proche du modèle européen. Les company towns américaines sont aussi créées et aménagées par des paternalistes et souvent très planifiées (Chicopee dans l'État de Géorgie, Vandergrift en Pennsylvanie, Arvida au Canada dans la province de Québec), mais elles peuvent aussi être plus spontanées, moins organisées et moins soumises à l'influence des patrons (Bagdad dans l'Arizona) voire quasiment pas (Anyox au Canada, en Colombie-Britannique). Il y a une grande porosité entre les modèles américain et européen pour de multiples raisons : lien étroit entre les deux continents, influence des penseurs européens, exemples des réalisations précoces européennes. Le modèle européen s'exporte partout en Europe et au-delà, de manière anisotrope, pour former des villes-usines à part: "[les grandes puissances européennes] exportent le village ouvrier ou la ville-usine, qui acquièrent, sous d'autres cieux, des traits physionomiques particuliers » (ibid., p. 9).

2 De ce fait, poser des regards croisés sur les modèles européens et nord-américains permet d'étoffer notre compréhension des villes-usines européennes. Celles-ci ont un intérêt pour la reconversion des anciennes villes-usines et le "redéveloppement territorial» (Daviet et al. 2006) des anciens territoires industrialo-urbains qu'elles constituent car elles ont une influence sur son potentiel post-industriel (Wirth et al. 2012) et, notamment, sur ses éléments patrimoniaux utiles au redéveloppement territorial : « il faut tenir compte du passé et de la mémoire d'un territoire afin de déterminer ses forces et ses faiblesses structurelles héritées dans l'objectif de le redévelopper» (Picon 2019, p. 50). Mais étudier la ville-usine, son déclin et son redéveloppement, nécessite aussi de mobiliser une kyrielle de concepts et de notions de géographie urbaine, et revient donc à faire un double travail de synthèse, non exhaustif et ouvert, sur les variations du modèle dans le monde occidental d'une part, sur la morphologie et la morphogenèse urbaines particulières de ces villes d'autre part. À partir de cela, nous ouvrirons quelques pistes de réflexions sur le redéveloppement territorial, sorte de transition maîtrisée, des territoires formés par les anciennes villes-usines occidentales.

\section{Le modèle de la ville-usine et ses nuances}

L'expression "ville-usine» est employée avec de nombreuses acceptions dans la littérature. D'autres expressions moins précises sont parfois employées pour qualifier de telles villes: "villes industrielles", "cités ouvrières ». Dans les publications de géographes francophones, on retrouve de nombreuses occurrences «villeusine » (Picon 2019 ; Edelblutte 2010a, 2010b, 2012, 2016; Del Biondo 2014, 2016; Luxembourg 2014 ; Jalabert et Grégoris 1987 ; Bruyelle Dézert 1983; Doyen 1983) qui font parfois la confusion entre villle-usine et ville industrielle. Une définition intéressante de la ville-usine a été faite par S. Edelblutte et complétée par une modélisation plus éloquente qu'une définition textuelle (figure 1 ) : «La ville-usine peut ainsi être considérée comme une ville entièrement ou presque entièrement née d'une ou plusieurs usines et/ou mines » (Edelblutte 2009). Cette définition a l'avantage de subsumer la diversité des villes-usines en intégrant toutes ses variations de taille, de genèse, d'organisation socio-spatiale et de situation. S. Edelblutte représente tous les éléments spatialisés du paternalisme industriel dans son schéma de la ville-usine : la composante 
productive (usines) et ses infrastructures ad hoc; l'habitat ouvrier, l'habitat patronal, les bâtiments économiques et sociaux, tout ce qui maintient la population sur place ; les éléments naturels à l'origine de la ville-usine, ceux qu'É. Reclus décrit dans la citation introductive ; les liens avec l'extérieur permettant d'acheminer les matières premières ainsi que les ressources humaines et d'exporter les marchandises. Enfin, S. Edelblutte ajoute que ces villes sont soit planifiées, issues de la volonté d'un seul industriel, soit non-planifiées, liées à l'agglomérat de plusieurs systèmes industrialo-paternalistes (Del Biondo et Edelblutte 2016). La définition de S. Edelblutte synthétise la plupart des publications francophones à ce sujet. Aussi, nous allons nous appuyer sur sa définition afin de mener une réflexion sur le modèle de la ville-usine et sur ce qui influence son organisation spatiale.

Figure 1 : le schéma type de la ville-usine (Edelblutte 2014)

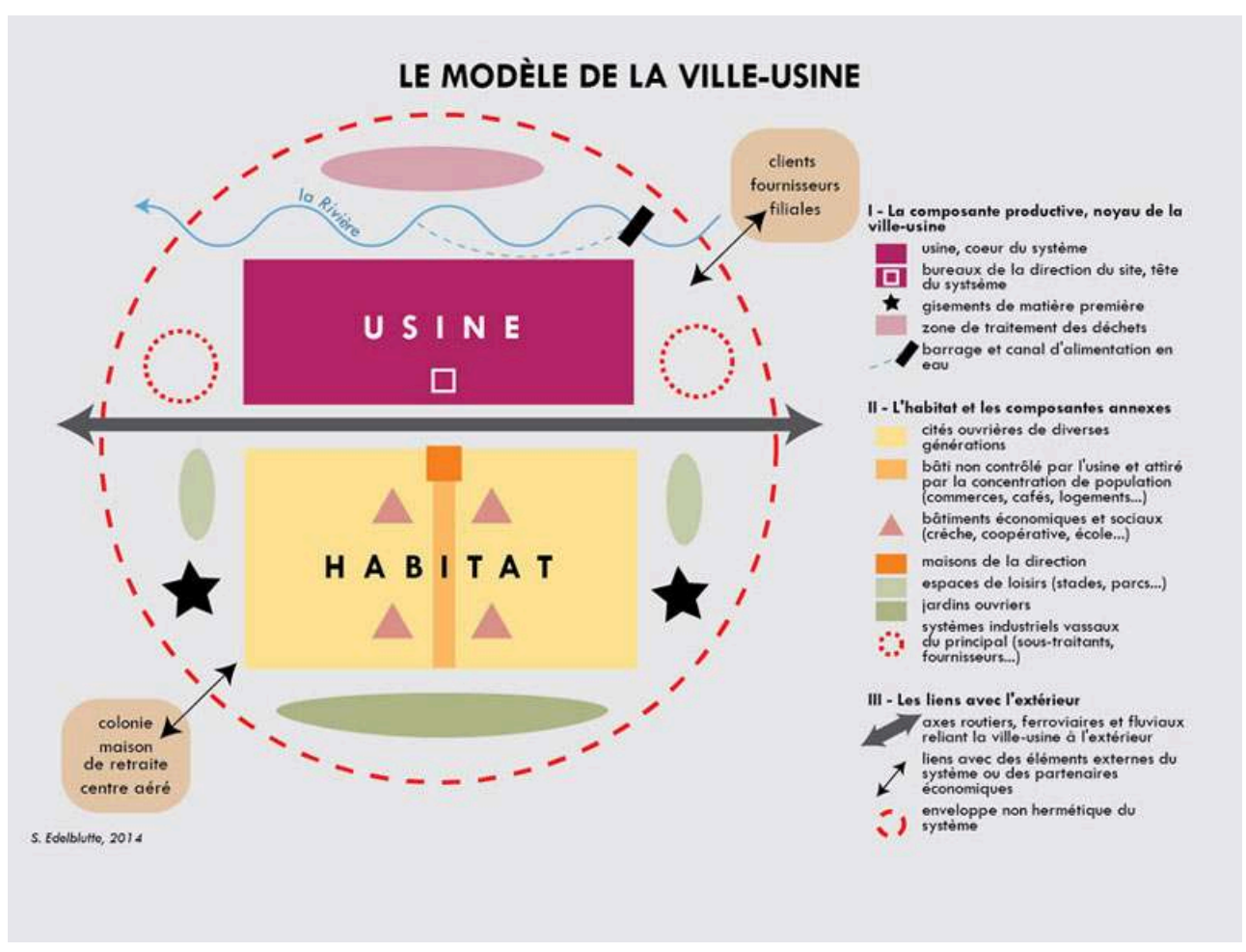

\section{La taille ou l'urbanité ? Différencier villes-usines, villages-usines et isolats industriels}

4 L'expression ville-usine a une double connotation associée à la notion de ville : elle peut faire penser que la ville-usine est grande et urbaine. En réalité, l'urbanité et la taille des villes-usines sont très variables, donnant à juste titre naissance à des appellations différentes selon les auteurs.

5 F. Schwarz parle d'« isolat industriel » en s'inspirant de P. Fluck pour « rendre compte du déploiement, à l'échelle d'un hameau, d'un géosystème industriel plus étoffé» (Schwarz 2019, p. 257). Il voit trois spécificités à l'isolat (ibid., p. 258 et 259) : l'isolement ; une genèse liée à une seule initiative patronale et à une mono-activité (c'est potentiellement le cas de toutes les nuances du modèle) ; une tendance au fonctionnement autonome (idem). Ces deux dernières caractéristiques sont relevées par tous les auteurs cités dans notre 
article, pour tous les géosystèmes industriels de ce type, quelles que soient leurs tailles, elles ne sont pas propres à l'isolat. Les caractéristiques particulières de l'isolat semblent plutôt être : sa localisation dans les espaces interstitiels et son isolement ; sa très petite taille et la faible densité des services qu'il offre. L'exemple de Trougemont (commune de Basse-sur-le-Rupt, Vosges) semble correspondre à un modèle situé entre l'isolat et le village-usine (figure 2).

Figure 2 : Le village-usine textile de Trougemont dans les Vosges (Picon 2019)

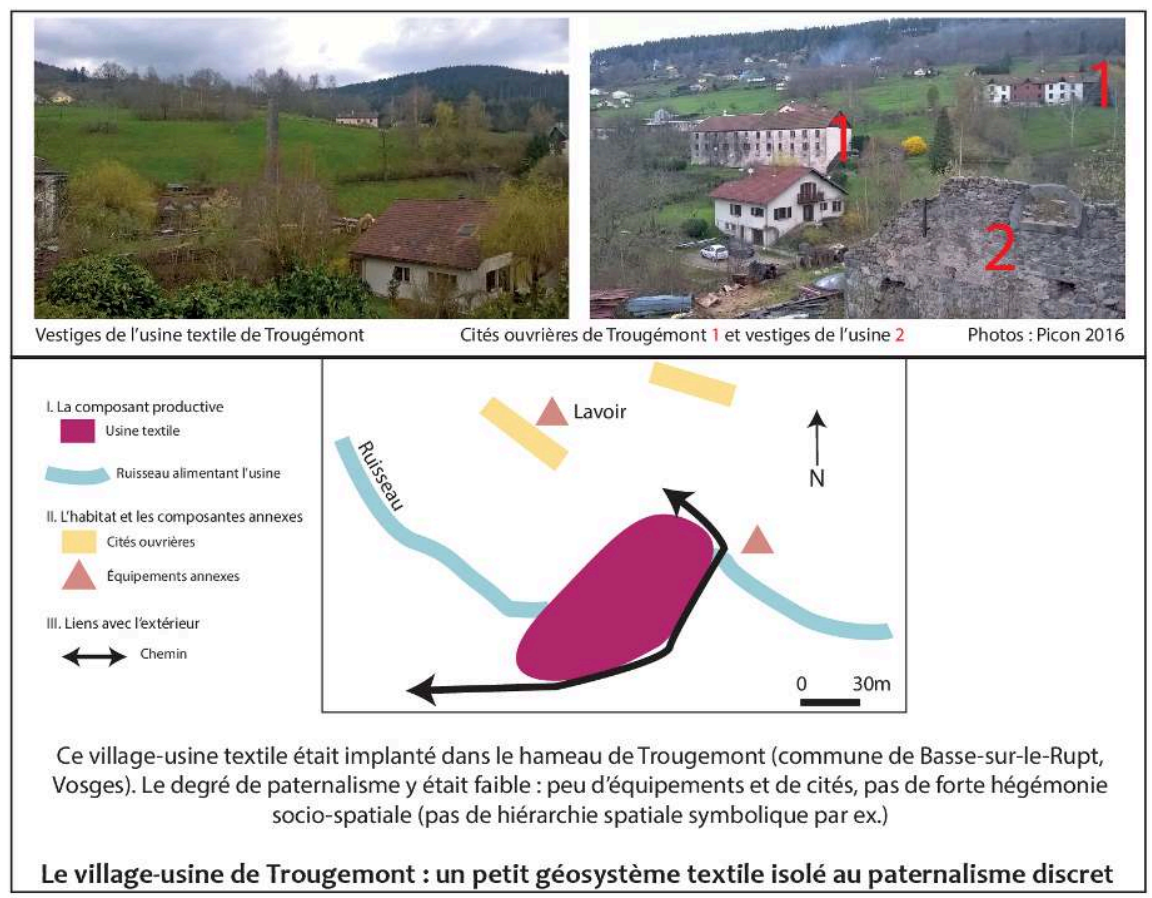

$6 \quad$ Le village-usine (Edelblutte et Legrand 2012) ou le village ouvrier (G. Dorel-Ferré 2016) désigne un ensemble plus complet que l'isolat industriel, mais de taille encore réduite. À la différence d'autres implantations industrielles rurales proto-industrielles (Edelblutte et Legrand 2012, Nacé et Nacé 2008), les villages-usines sont essentiellement voués à l'industrie : «Pour la première fois, en effet, apparaissent des villages créés de toutes pièces consacrés à une monoactivité qui n'est pas agricole »(Dorel-Ferré et Bergeron $1996 \mathrm{p}$. 60). Il existe une grande variété de villages-usines. Leurs caractéristiques les plus communes sont les suivantes : ils sont fondés pour et par une industrie monosectorielle ex nihilo, ils sont implantés en milieu rural, ils n'ont pas la capacité d'urbaniser leur territoire d'influence, ils sont à une échelle plus grande que celle de la ville (ce critère varie donc selon les pays), ils sont rarement concentrés, plutôt diffus. Les plus petits systèmes sont minimalistes et les éléments spatiaux du paternalisme y sont rares, mais pas autant que dans l'isolat industriel.

7 Les modèles les plus paternalistes fonctionnent souvent comme de petites villes-usines planifiées (Crespi d'Adda, figure 3). 
Figure 3 : Crespi d'Adda, une ville-usine paternaliste planifié (Picon 2019)

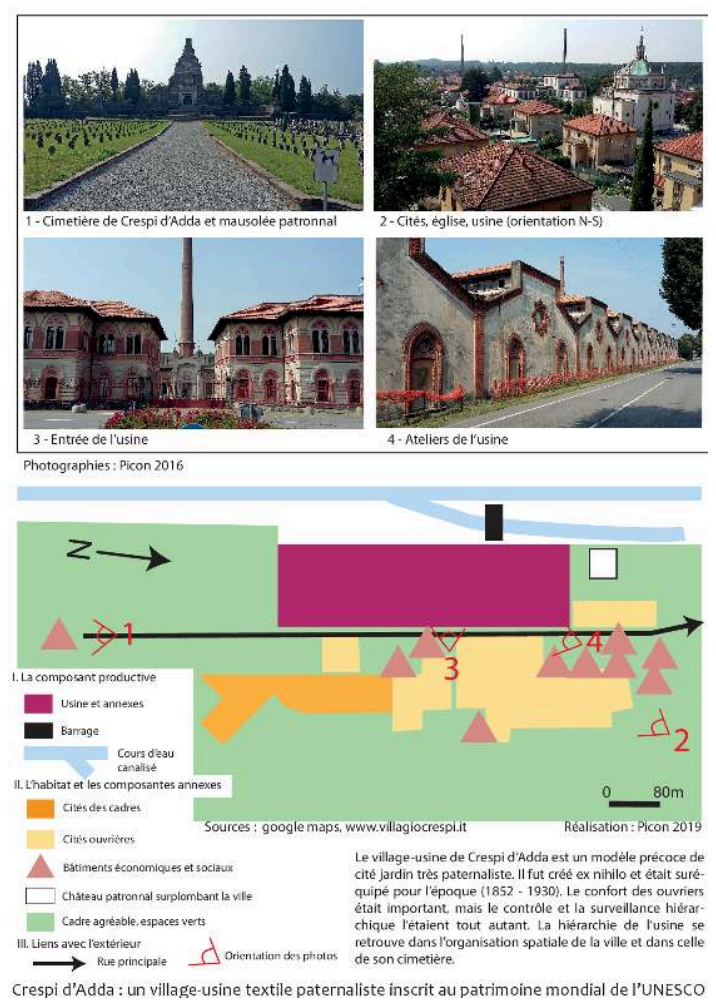

8 La limite entre ville et village-usine est ainsi souvent confuse. Cela est sans doute dû à l'image plus ou moins urbaine que renvoient certaines industries et certains bâtiments industriels. Le textile ancien renvoie plus souvent à une image de ruralité et est considéré, à tort, comme peu polluant. Les immeubles-machines et les usines tubulaires de l'industrie lourde et des industries extractives renvoient à une image plus sale et urbaine. Enfin, les usines contemporaines cachées dans des «hangars fonctionnalistes passe-partout» (Edelblutte 2012 p. 11) semblent se fondre aussi bien dans des espaces urbains que dans des espaces ruraux, car, paradoxalement, ces bâtiments adaptés à leur fonction occultent tout à fait cette dernière. Cette confusion est aussi due à la taille moyenne de la plupart des villes-usines et à la fragilité de leur urbanité, dépendante de l'industrie : manque de diversité des activités économiques, taille et extension limitées par les complexes industriels, éclatement urbain, etc. C. Luxembourg définit notamment la ville-usine en fonction du degré d'imbrication de l'industrie au tissu urbain de la ville : dans la ville-usine, l'industrialisation et l'urbanisation est centripète et (souvent) planifiée par et pour l'usine (Luxembourg 2008, pages 72 à 78). Dans le cas de la ville-usine, elle parle d'une "[...] situation de plus grande dépendance aux établissements de production qui [...] pose [...] la question de la pérennité urbaine. " (Luxembourg 2008, p. 77). Le cas de l'ancienne ville-usine de Hayange dans la vallée de la Fensch est assez emblématique de toutes ces problématiques (figure 4).

9 La porosité des deux modèles provient aussi du caractère urbain partiel des villagesusines: la population est souvent trop peu nombreuse, mais le peuplement et l'urbanisation sont denses et une certaine diversité peut être générée par l'usine (commerces, services, etc.). Enfin, le cadre rural dans lequel sont implantées la majorité des villes-usines, du fait de l'influence des idées hygiénistes, accentue également cette confusion entre les deux modèles. Le village-usine est donc, de manière simplifiée, soit 
une ville-usine plus petite, soit une ville-usine perçue comme plus petite. L'isolat, plus marginal dans les publications, semble désigner un type bien particulier de villageusine très isolé. En somme, le critère de taille ne permet pas d'exclure une réalisation de la liste des villes-usines, seulement d'en étendre le spectre. La question de l'urbanité est sans doute plus centrale, car la carence en urbanité est commune à presque toutes ces réalisations.

\section{La genèse}

La genèse de la ville-usine est liée à l'industrie mono-sectorielle et à la présence d'une ou de quelques entreprises industrielles plutôt qu'à l'entreprise unique (les anglophones qualifient les villes industrielles monosectorielles de corporation town ou d' industrial city). Cependant, s'il est courant que des villes-usines européennes soient sous l'influence de plusieurs industriels, leur genèse est souvent due à une seule entreprise. En effet, pour générer de tels systèmes industriels, il faut avoir la maîtrise de l'espace. L'industrie principale attire en général des "industries vassales" comme l'indique S. Edelblutte dans son schéma de la ville-usine (figure 1). Ce fut, par exemple, le cas des villes-usines sidérurgiques du Pays Haut lorrain.

11 Les limites administratives et les administrations des communes françaises sont souvent peu adaptées aux besoins des patrons paternalistes. Pour y remédier, les patrons s'impliquent souvent dans la vie politique locale: «la liste des industriels $d u$ XIXème siècle s'investissant dans la politique locale est impressionnante 》(S. Edelblutte 2010 p. 3). De plus, ils modifient ou créent de nouveaux territoires politiques afin de les adapter au territoire fonctionnel de la ville-usine (ce fut le cas à Hayange, $c f$. figure 4) : "pour les entrepreneurs, il est essentiel que l'usine soit sur une seule commune ou sur le moins de communes possible pour en faciliter la gestion. La seule solution, avant la mise en place d'intercommunalités efficaces à la fin du XXème siècle, est la modification des territoires communaux, adaptant les territoires politiques aux territoires fonctionnels» (ibid., p. 9). Leur genèse est alors le fruit de plusieurs créations ex nihilo ou presque. Enfin, lorsque la création n'est pas purement ex nihilo, elle est souvent le fruit du passage de la protoindustrie à l'industrie lourde, et donc de la métamorphose d'un petit bourg proto industriel en ville-usine dédiée à l'industrie lourde (Moyeuvre-Grande dans la vallée de l'Orne, Hayange dans la vallée de la Fensch, cf. figure 4). 
Figure 4 : Hayange, une ex ville-usine au territoire éclaté et coupé en deux (cités au sud et au Nord sur les versant de vallée et sur les plateaux, usines dans le fond de vallée ; Picon 2019)

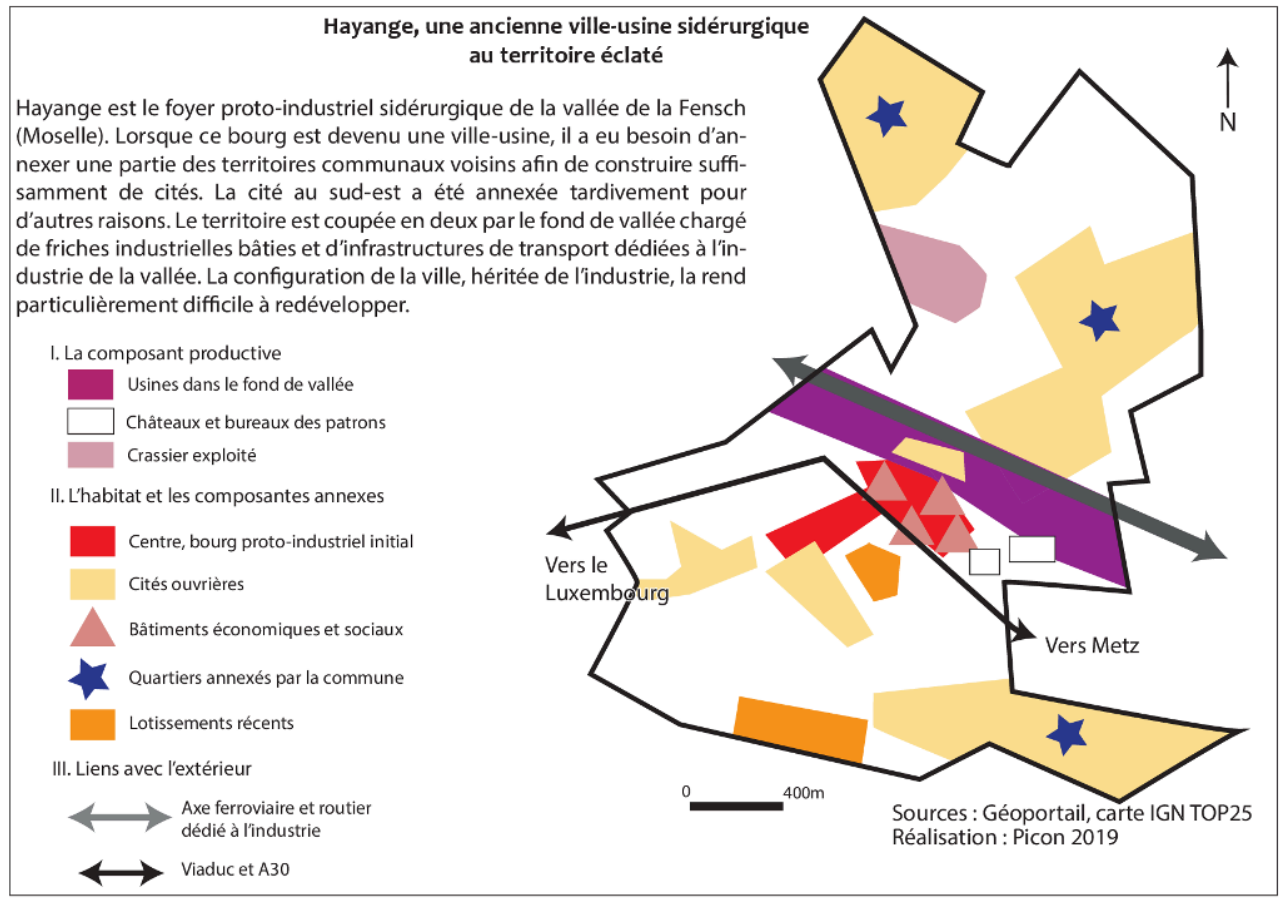

12 On peut, de plus, distinguer : des villes fondées sur l'exploitation d'une nouvelle ressource qui était soit inconnue, soit impossible à extraire avec des techniques anciennes, que l'on peut appeler sobrement villes-usines extractives; des villes fondées sur une politique sociale novatrice du patron (social moulding, c'est-à-dire modelage ou façonnage social selon J. D. Porteus (1970, p. 130), qui sont dans la plupart des cas des villes-usines manufacturières (la ville-usine textile de Crespi d'Adda en est un exemple parfait, $c f$. figure 3) ; et puis des villes-usines extractives et manufacturières ou mixtes (les villes de la sidérurgie du bassin ferrifère du Pays Haut lorrain par exemple) qui ont un fonctionnement, contraint, assez proche des villes-usines purement manufacturières décrites par J. D. Porteus.

13 La genèse des villes-usines est essentielle pour comprendre leur morphogenèse urbaine et, par extension, leur morphologie actuelle. C'est donc un des éléments qui doit être étudié systématiquement pour diagnostiquer et redévelopper des territoires villeusiniers. Toutefois, ce n'est pas le seul élément à avoir une influence sur l'organisation et la planification de la ville. Sur ce point, l'intensité du paternalisme industriel joue un rôle majeur.

\section{L'organisation socio-spatiale : influence du paternalisme industriel}

14 «En Angleterre, de rares industriels philanthropes - ou des patrons très avisés - ont compris qu'il n'y a qu'un moyen de combattre cette tendance haineuse à l'avilissement du travail [...] On peut citer, parmi ces réalisations patronales, les « villes-jardins » ou garden cities, qui contrastent merveilleusement par leur beauté architecturale, leur hygiène et leur confort avec les fumeuses cités voisines " (Reclus 1905, p. 347). Sous l'influence des idées hygiénistes, on implante souvent les company towns et les villesusines paternalistes dans un cadre rural ou agréable (cf. figure 5). Associé à la présence 
de jardins potagers qu'il est bien vu d'entretenir, ce cadre est choisi afin de donner aux ouvriers une mentalité conservatrice de petit propriétaire terrien : «[...] the workers' economic and emotional commitment to home ownership would foster a concern for preserving property values, a sense of place, and a sense of identification with the company " (Mosher 1995, p. 103). Une bonne moralité étant synonyme d'une bonne capacité de travail selon les préceptes du catholicisme social : « [...] good people made good workers [...], resident-workers would give their allegiance to those who made it possible for them to live in quality environments at low cost » (ibid., p. 91).

Figure 5 : Chicopee dans l'État de Géorgie, une ville-usine textile moderne (Picon 2019)

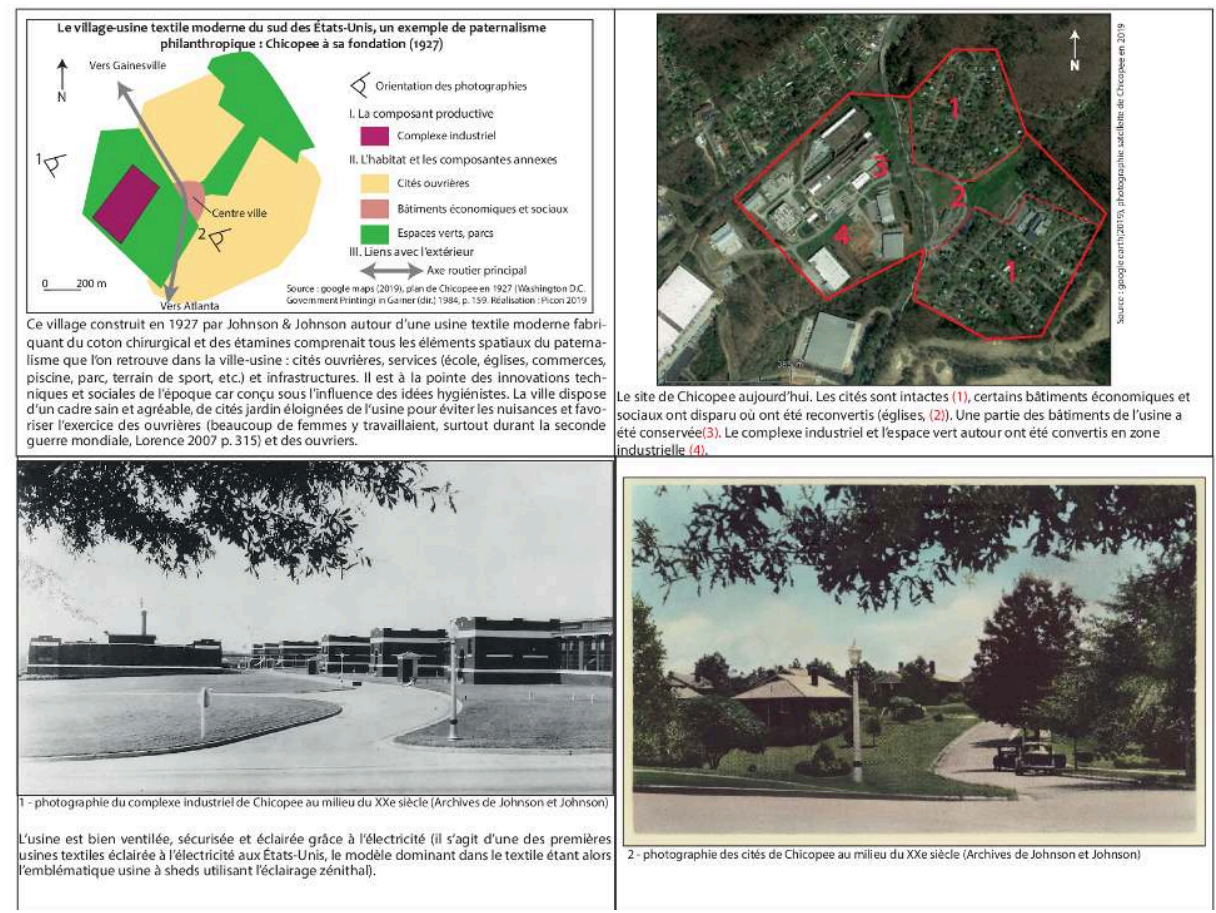

On a donc ici deux éléments spatiaux participant à l'acceptation de l'ordre social paternaliste : la localisation (rurale et agréable) et les jardins (ouvriers ou de cité). De plus, l'ensemble des bâtiments économiques et sociaux, des infrastructures de loisirs ainsi que l'habitat de la ville-usine paternaliste, ceux représentés sur le schéma de S. Edelblutte (figure 1), participent également à l'accord spontané de même que la composante productive qui fournit travail et salaire (cf. III.B.).

Ces éléments (bâtiments, aménagements) fonctionnent pour toutes les villes-usines paternalistes, qu'elles soient planifiées ou non. Celles qui sont planifiées peuvent encore renforcer cet accord "spontané » par leur organisation spatiale hiérarchisée : "Le paternalisme est une pure libéralité aux antipodes de l'obligation. Même s'il implique des devoirs de la part du patron, le paternalisme est autoritaire et hiérarchique » (A. Gueslin 1992, p. 202). Cela se retrouve dans l'habitat qui est organisé suivant une "hiérarchie et une relative homogénéité" (Dorel-Ferré 2016, p. 7). Cette hiérarchisation de l'espace paternaliste a deux fonctions : accord "spontané » des population et coercition ( $c f$. III.B.). L'exemple de Jœuf (Meurthe-et-Moselle) illustre bien ce phénomène (figure 6). 
Figure 6 : la hiérarchisation symbolique de l'espace jovicien (Picon 2019)

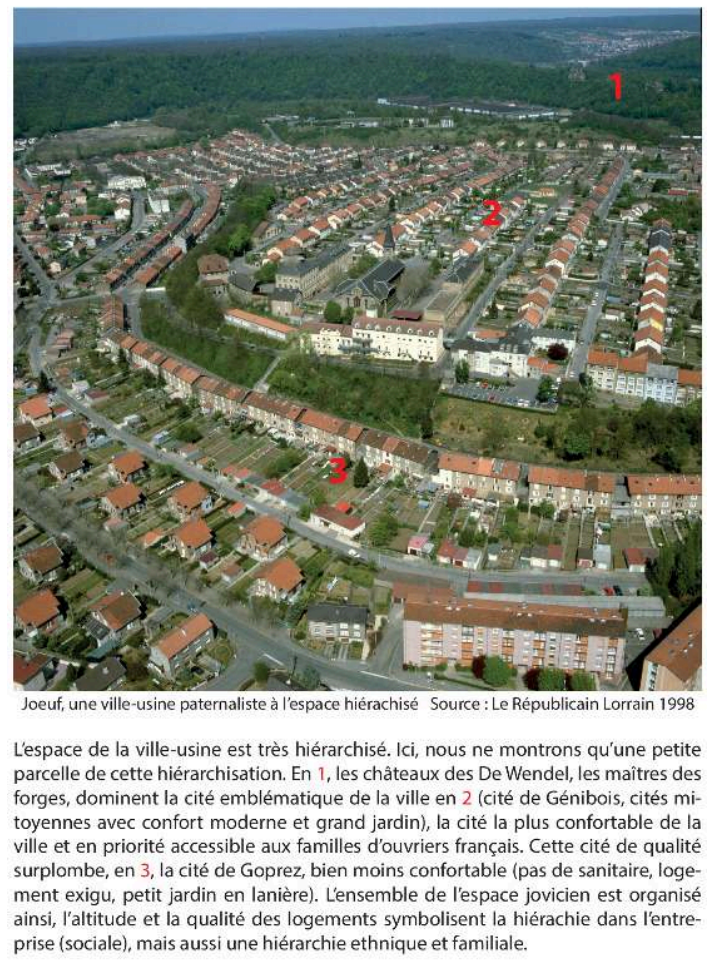

17 La hiérarchie dans l'espace paternaliste se retrouve à deux niveaux, le premier niveau consiste en une assignation de l'espace selon différents critères de hiérarchisation: l'accès aux différentes qualités de cités est permis en fonction de la position dans l'entreprise, de la nationalité, de la situation familiale et du genre. En effet, selon les préceptes des idéologues associés au paternalisme : «[...] les jeunes gens ne se soumettent point aux efforts qu'impose l'acquisition préalable du foyer, et ils ne s'habituent pas, dès le début de leur vie, aux autres pratiques essentielles, si le sacrement du mariage ne préside pas à l'organisation de la société entière. En cette matière, l'accord de la loi religieuse et de la loi civile a été l'un des fondements de toutes les sociétés prospères [...] " (Le Play 1870, p. 161). Ce contrôle se traduit spatialement par une assignation de l'espace liée à cette hiérarchisation de la situation familiale : «Les villes ouvrières et les villages, lorsqu'ils ont logé des hommes célibataires, les ont accueillis dans des hôtels spécifiques, voire dans des auberges, sans les isoler totalement" (Dorel-Ferré 2016, p. 9). Lorsqu'il s'agissait de manufactures à personnel exclusivement féminin, "une main d'œuvre féminine autonome parce que salariée est vite apparue comme une menace pour la stabilité de la société patriarcale» (ibid., p. 9) et il est apparu nécessaire aux industriels de contrôler cette population: vie dans des pensions, tutelle religieuse. La pratique dominante reste toutefois d'accueillir des familles entières dans des cités ouvrières, car c'est un gage de stabilité pour les patrons.

18 Le deuxième niveau de cette hiérarchie a deux fonctions : symbolique et de contrôle. La hiérarchie de l'entreprise est mise en scène dans l'espace. Par exemple, les châteaux patronaux sont souvent placés au-dessus des cités ouvrières (figure 6). De ce jeu de dénivellations, naissent un sentiment de surveillance a minima et une surveillance bien réelle a maxima. En effet, dans les cas les plus poussés, l'aménagement de l'espace est prévu afin d'assurer une intervisibilité maximale et un autocontrôle tacite de la 
population. Par exemple, à très grande échelle, les jardins des cités sont intervisibles, celui qui entretient mal son potager est mal perçu par la communauté ouvrière (autocontrôle). Les cités ouvrières les plus hautes dans la hiérarchie sont parfois positionnées afin de surveiller celle des ouvriers moins bien perçus (immigrés par exemple) dans les cités moins qualitatives. A. Gueslin pressent cette dimension du contrôle paternaliste lorsqu'il écrit : "Il y a une discipline du travail et on ne peut rejeter l'approche foucaldienne [...]. «Surveiller et punir » pour produire mieux, voilà une dimension du projet paternaliste qui mériterait d'être mieux analysée [...]»(Gueslin 1992, p. 209). A. Gueslin n'est pas le seul à analyser la ville-usine avec des outils foucaldiens, certains sociologues voient dans sa version idéelle une "ville panoptique» (Murard et Zylbermann 1977, p. 23), c'est-à-dire que le contrôle s'y exerce essentiellement par la surveillance. Ce contrôle se retrouve exacerbé dans les mill towns textiles du sud des États-Unis (figure 7). Et, en effet, lorsque Foucault a conceptualisé la "surveillance » hiérarchique, il a notamment pensé aux villes des paternalistes (et à leurs usines) : «Le camp, c'est le diagramme d'un pouvoir qui agit par l'effet d'une visibilité générale. Longtemps on retrouvera dans l'urbanisme, dans la construction des cités ouvrières [...], ce modèle du camp ou du moins le modèle qui le sous-tend: l'emboitement spatial des surveillances hiérarchisées". (Foucault 2008, p. 202). M. Foucault associe également cette idée à Arc-et-Senans ${ }^{1}$ et son architecture circulaire : "au centre [...] une haute construction devait cumuler les fonctions administratives de direction, policières de surveillance, économiques de contrôle et de vérification, religieuses d'encouragement à l'obéissance au travail»(ibid., p. 204). Les exemples se prêtant à une lecture foucaldienne de l'espace ville-usinier semblent nombreux : le plan type du cotton mill village dans le sud des Etats-Unis (figure 1), Crespi d'Adda (figure 3), Jœuf (figure 6), les cités de Gargan à Hayange (figure 4, les maisons des cadres y surplombent les cités du sud-ouest de la ville).

Le degré de paternalisme est souvent corrélé au type de ville-usine (extractive ou manufacturière comme le souligne J.-D. Porteus), mais aussi à sa situation plus ou moins marginale, plus ou moins isolée. 
Figure 7 : schéma type du village-usine textile américain du XIXe siècle (Picon 2019)

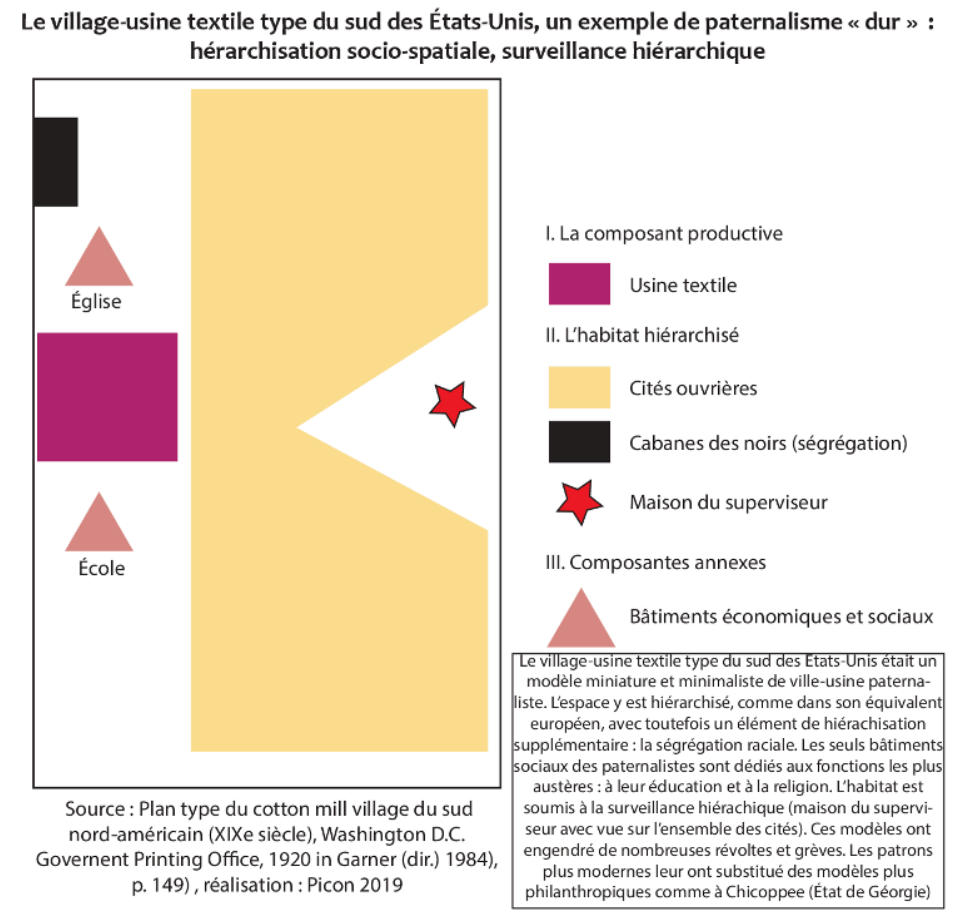

\section{La situation et le cadre}

Nous l'avons vu avec le cas des isolats industriels évoqués par F. Schwarz, la situation (au sens localisation à petite échelle) des villes-usines génère une grande variété de modèles. En revanche, la ville-usine est presque systématiquement implantée dans un cadre rural (ou vierge comme nous le verrons pour l'Amérique du Nord), avec parfois un caractère proto-industriel, pour différentes raisons: hygiénisme, paternalisme, foncier disponible.

Les villes-usines sont souvent isolées lors de leur création ou situées dans des marges ou des espaces interstitiels. Les plus isolées forment des isolats, celles qui restent éloignées des territoires urbains et qui n'ont pas la force d'attraction suffisante pour urbaniser leur territoire adjacent forment souvent des villages-usines ou des villesusines. Celles qui sont concentrées dans des vallées forment des territoires particuliers. C'est le cas, notamment, des villes-usines du Pays Haut lorrain. Isolées au départ, mais proches du fait de leurs implantations à proximité de deux ressources clefs (l'eau et le fer), elles forment progressivement de vastes conurbations linéaires: des vallées industrielles. Dans la deuxième moitié du XXe siècle, la coalescence des villes-usines sidérurgiques du Pays Haut n'est plus seulement physique car les entreprises et leurs installations se réorganisent pour former de vastes usines intégrées qui occupent tout ou presque les fonds de vallée (certaines usines restent indépendantes toutefois). Des villes-usines plus isolées (dans des territoires où l'industrialisation et l'urbanisation sont diffuses et discontinues) telles que la Verrerie-de-Portieux (Vosges) pour les modèles anciens et manufacturiers ou Stiring-Wendel (Moselle) pour les modèles anciens et extractifs-prospectifs, restent isolées et sous la tutelle d'une seule entreprise jusqu'à leur déclin. Les villes-usines extractives et prospectives ont souvent la particularité d'être situées dans des marges (frontières ou limites de bassins miniers 
connus) au moment de leur création du fait de leur dimension prospective. Ces dernières ont toutes un toponyme particulier (il contient le nom du patron ou de l'entreprise) car ce sont soit des créations communales, soit des territoires fonctionnels à part et isolés dans une commune (la Verrerie-de-Portieux).

La situation des villes-usines influence ses autres caractéristiques et son degré de planification. Si une ville-usine est extractive et isolée, elle peut potentiellement être moins planifiée du fait des contraintes et du caractère temporaire de l'exploitation minière. L'isolement, lui, induit souvent un paternalisme plus souvent de nécessité et de coercition que de confort, car un territoire isolé a d'un côté peu d'intérêt pour soigner l'image d'une entreprise ( $a u X X^{e}$ et au début du XXe siècle) et a, de l'autre, besoin d'équipements pour assurer une certaine autonomie et une certaine attractivité à la population ouvrière locale. L'isolement de ces territoires est très variable selon le pays concerné, il peut être absolu (Sibérie, Nord canadien : villes frontières) ou relatif (Europe de l'ouest, Europe centrale, Etats-Unis : espaces interstitiels).

Il existe ainsi des villes-usines planifiées et non planifiées (Del Biondo et Edeblutte 2016). La taille, la morphogenèse urbaine, la situation et l'aménagement paternaliste de l'espace influencent l'organisation spatiale de la ville-usine et font qu'elle est plus ou moins planifiée, qu'elle a une qualité urbaine plus ou moins grande, et, finalement, que son territoire est plus ou moins facile à redévelopper. Les villes-usines spécifiques d'Amérique du Nord, fortement influencées par les modèles et par les utopies et réalisations européennes, permettent encore mieux d'apprécier les caractéristiques des villes-usines présentant un intérêt praxéologique pour le redéveloppement des territoires ville-usiniers.

\section{Les apports des company towns au modèle de la ville- usine : des modèles nord-américains singuliers qui résonnent avec les modèles ouest-européens}

Les premiers modèles industriels d'Amérique du Nord, des mill villages textiles, naissent dans un contexte économique et géopolitique particulier, l'embargo act de Thomas Jefferson (1807 - 1809) interdisant les entrées et sorties des navires étrangers sur son territoire, et donc bloquant toutes les importations maritimes: "Mill villages of the textile industry sprang up throughtout New England during the half of the nineteenth century, in part throught the efforts of [...] proponents of industrialism [...] as a result of Jefferson's Trade embargo against England » (Garner 1992 p. 11). Le modèle américain du mill village ou de company village correspond sans surprise à celui du village-usine européen avec ses spécificités et une certaine austérité (figure 7). Les modèles plus récents sont souvent plus philanthropes et paternalistes (figure 5, Chicopee), organisés "like a family" (Garner 1984, p. 143).

\section{Une grande diversité de modèles}

À une échelle plus petite, il existe une mosaïque de modèles. On trouve plusieurs expressions proches de «ville-usine » telles que company-town, factory town et mill town. La mill town est moins présente que la company town dans les publications récentes des géographes. L'expression est mal définie, mais on peut la résumer ainsi : elle désigne les 
villes-usines fondées par une ou plusieurs entreprises. Elle sert le plus souvent à désigner, de fait, des company towns. Dans les publications des géographes, historiens et sociologues, mill town renvoie souvent au premières villes textiles étasuniennes et britanniques (Byington 1909; Porteus 1970; Garner 1984 et 1992; Mullin 1998; Colocousis 2012 ; Hodges et Frank 2014). Le fait que mill désigne d'abord l'usine protoindustrielle par excellence (le moulin) explique sans doute que mill town renvoie à des systèmes industriels précoces. Factory town est plus rare dans les publications universitaires. L'expression est soit utilisée comme traduction anglaise du modèle français de ville-usine, soit comme synonyme de mill town. Les deux mots ont des sens très proches et leurs usages dans la littérature ne nous permettent pas de différencier mill town et factory town. L'expression model town désigne au départ les villes-usines paternalistes et planifiées du Royaume-Uni. Elle a finalement pris un sens plus général et désigne toutes les villes-usines de ce type. «Model » s'applique, en anglais, à toutes les déclinaisons de la ville-usine (factory, mill, ou industrial town). Le modèle le plus commun et détaillé dans la littérature est donc celui de company town. L'expression désigne en réalité plusieurs nuances d'un même modèle que certaines caractéristiques singularisent de la ville-usine européenne.

Depuis plusieurs décennies, l'expression company town désigne essentiellement des villes fondées et contrôlées par une seule entreprise : "A company town is a settlement built and operated by a single business enterprise » (Garner 1992, p. 4). D'après Garner, c'est même une condition pour qu'un mill village devienne une company town: "[...] mill villages could develop into company towns if in time a single enterprise prevail» (ibid., p.4). La définition se borne aussi aux villes gérées par une seule entreprise, alors que celle de la ville-usine est liée à l'industrie mono-sectorielle et à la présence d'une ou de quelques entreprises industrielles plutôt qu'à l'entreprise unique.

Les autres différences avec la ville-usine européenne tiennent à l'origine du modèle. Il ne concernait à l'origine que les villes-usines d'Amérique du Nord (majoritairement extractives) et avait une connotation péjorative dans ses premières expressions. "Les "company towns» [...] correspondent à une situation économique particulière (les grandes entreprises privées) dans un cadre chronologique précis [...] dans un espace précis où il a tout son sens: les États-Unis et éventuellement le Canada." (Dorel-Ferré 2016, p. 8). Cette définition qui place la company town en Amérique du Nord corrobore en partie celle de J. S. Garner qui considère que l'expression a été inventée aux États-Unis à la fin du XIXe siècle pour d'abord désigner, avec une connotation péjorative, des villes-usines extractives, sidérurgiques, ou manufacturières (textile, papier, etc.) du XIXe voire du XVIIIe siècle, mais que l'expression désigne désormais autant les villes-usines nordaméricaines qu'européennes (J. S. Garner - dir. 1992, p. 3). Ces premières company towns sont soit planifiées en amont (model town), soit plus spontanées, et leur évolution va vers la planification (Garner 1984, p. 5 ; Mosher 1995, p. 85).

\section{Marginalité et isolement des villes de compagnie}

Pour compléter cette définition de la company town, il est nécessaire de regarder vers le Canada. La plus commune des définitions de la company town reste celle, géographique, qui se restreint aux company towns d'Amérique du Nord. Pour cette raison, nous préférons, comme L. K. Morisset, l'anglicisme «ville de compagnie» à «ville d'entreprise » car elle renvoie spécifiquement au modèle américain plutôt qu'à tous les 
modèles de villes-usines mono-entreprise et est donc plus fidèle au sens de l'expression originelle. L. K. Morisset redéfinit récemment le modèle de la company town: "Par « villes de compagnie " nous entendons [...] des ensembles mono-industriels planifiés, où une entreprise a fait construire des habitations pour ses travailleurs, généralement sur un plan urbain, et mis en place divers équipements. À cette définition générique, qui leur a valu d'être qualifiées de "villes industrielles planifiées " et, plus souvent, de single-enterprise communities, les villes de compagnie ajoutent cette caractéristique d'être des frontier towns " et "ce sont principalement des "villes du secteur primaire ", des resource towns " (Morisset 2017, p. 6). Enfin, une dernière caractéristique de ces company towns canadiennes, c'est leur isolement originel extrême : "elles s'affirment comme le résultat de leur éloignement, lequel aurait commandé, pour que l'industrie y survive, de tout créer, du parcellaire aux églises». On retrouve ici les éléments spatiaux du paternalisme industriel que S. Edelblutte décrit dans son modèle de la ville-usine européenne (cf. figure 1). Cependant, leur construction s'explique en partie, dans le cas canadien, par l'extrême isolement des villes de compagnie extractives, alors que dans le cas européen c'est le contexte rural et les idées paternalistes qui en sont plutôt à l'origine. Dans les deux cas, c'est la nécessité pragmatique d'attirer et de maintenir une population ouvrière sur place qui prévaut. C'est également cette différence dans le degré d'isolement et de marginalité qui explique que les villes de compagnie canadiennes sont soit des villes neuves, pionnières, installées sur une terre encore inoccupée (Uranium City, Keno City); soit des villes de colonisation installées sur des terres occupées par les premiers peuples (Anyox au nord de l'actuel territoire des Nisga'a en Colombie-Britannique) : « the company town was widely uses in areas of white colonial settlement " (Porteus $1970 \mathrm{p}$. 129). Dans les deux cas, elles sont, avant leur création, sans limites administratives, sans administration, sans parcellaire. De plus, ces villes de compagnie sont souvent des villes-frontières (a minima pionnières) comme l'indique $\mathrm{L}$. $\mathrm{K}$. Morisset ou bien des villes permettant de conquérir des territoires neufs, selon un processus proche de celui du front pionnier de F. J. Turner, comme nous l'indique aussi J. D. Porteus lorsqu'il parle du contexte génésiaque des company towns : "As possibly unexplored, usually unexploited, territory, the company town was widely uses in areas of white colonial settlement. Essentially a frontier-style method organization, the form could perhaps be fitted into Turner's frontier model » (Porteus 1970 p. 129). Même si certaines villes-usines extractives d'Europe de l'ouest sont situées en marge (par leur " position géographique », et par leur "état social »; Bailly 1983 p. 74) et ont ces dimensions pionnière, exploratoire et donc potentiellement transitoire (Stiring-Wendel en Moselle, Jœuf en Meurthe-et-Moselle², figure 6), aucune n'a, comme certaines villes de compagnie canadienne, une fonction de front pionnier. Celles-ci permettaient, en effet, à l'État canadien de peupler, d'aménager, de s'approprier et de connecter au reste de son territoire un territoire encore " vierge »: ce sont, au moment de leur création, des espaces en marge. Ils le restent souvent. La plupart du temps, ces villes sont donc fondées par des entreprises d'État (crown corporations). Leur éloignement en fait des villes peu paternalistes sauf lorsqu'elles ont cette fonction pionnière. Lorsqu'elles ont essentiellement un but extractif, sans cette fonction pionnière, et donc un caractère transitoire, les patrons y logent plutôt une main d'œuvre masculine et ne mènent pas une politique de la famille paternaliste, car ils n'ont pas besoin de maintenir longtemps les populations sur place. Lorsqu'elles sont planifiées, c'est plus pour servir l'organisation du travail qu'une organisation paternaliste ou philanthropique. 
29 L'immensité et la faible densité de population du Canada (3,76 hab./ $\mathrm{km}^{2}$ au Canada en 2019, $113 \mathrm{hab} . / \mathrm{km}^{2}$ en France en 2018) font que l'isolement des villes de compagnie est sans commune mesure avec celui de n'importe quelle ville française. Cependant, la marginalité de certaines villes de compagnie pionnières nous permet aussi de penser autrement la ville-usine lorsque celle-ci se situe dans les marges sociales (après leur déclin), spatiales (frontières, espaces interstitiels) ou liées aux risques industriels (effondrements miniers, explosions, pollution, etc.). Quelques publications évoquent ces problématiques dans les villes industrielles, tant sur les espaces interstitiels (Férérol 2014), que sur les espaces transfrontaliers ou sous l'influence d'un espace transfrontalier (Del Biondo 2014 ; Picon 2019) et les risques industriels (Blésius 2014). Aucune, en revanche, ne traite précisément des questions de marginalité dans la villeusine; voilà qui ouvre quelques pistes de réflexions pour mieux penser son redéveloppement territorial.

\section{Au croisement des modèles européen et américain : apports des company towns récentes et du cas particulier d'Arvida}

Arvida, fondée en 1926 par l'Aluminium Company of America (Alcoa) non loin du Lac Saint-Jean au Québec (Canada), correspond à une singularité de ce modèle, une model company town tellement planifiée et tellement influencée par les utopies européennes que L. K. Morisset considère qu'elle dépasse même la fiction : "[...]particular industrial preconditions, like those Garnier himself imagined for his hydro-powered metallurgical city [la cité industrielle] [...] brought Arvida into being for the first and undoubtedly the last time in the history of cities, a particular conjunction of idealism, expertise, and exceptional geography allowed what remained only a dream in Europe to come to fruition in Arvida, the town where reality went beyond fiction " (Morisset 2011, p. 6). L'exemple d'Arvida est sans doute le plus emblématique de la porosité entre les modèles européens et nord-américains : c'est la réalisation la plus complète d'une utopie née en Europe (figure 8). La qualité et le degré des réalisations spatiales des paternalistes dépend aussi de leur expérience en matière d'aménagement du territoire, des qualités du site et de la situation des espaces à leur disposition et de l'idéalisme des patrons comme le rappelle L. K. Morisset avec le cas d'Arvida: "a particular conjunction of idealism, expertise and exceptional geography" (ibid., p. 6) ont permis à Arvida de devenir la "cité industrielle" du continent américain. L'exemple du patron paternaliste très progressiste de Chicopee (figure 5) semble aller aussi dans ce sens. Quoi qu'il en soit, le paternalisme est avant tout au service de la production industrielle et de l'organisation du travail, et cherche toujours à attirer et à maintenir les ouvriers sur place.

Figure 8 : plan lithographique d'Arvida (réalisation : Harry Brainerd et Hjalmar Skougor, 1925 ; source : Ville de Saguenay). Légende traduite (de haut en bas) : zone résidentielle, zone commerciale, zone industrielle, usine existante, usine future, affleurements rocheux, espaces verts et ravines ${ }^{3}$, bâtiments publics et institutionnels 


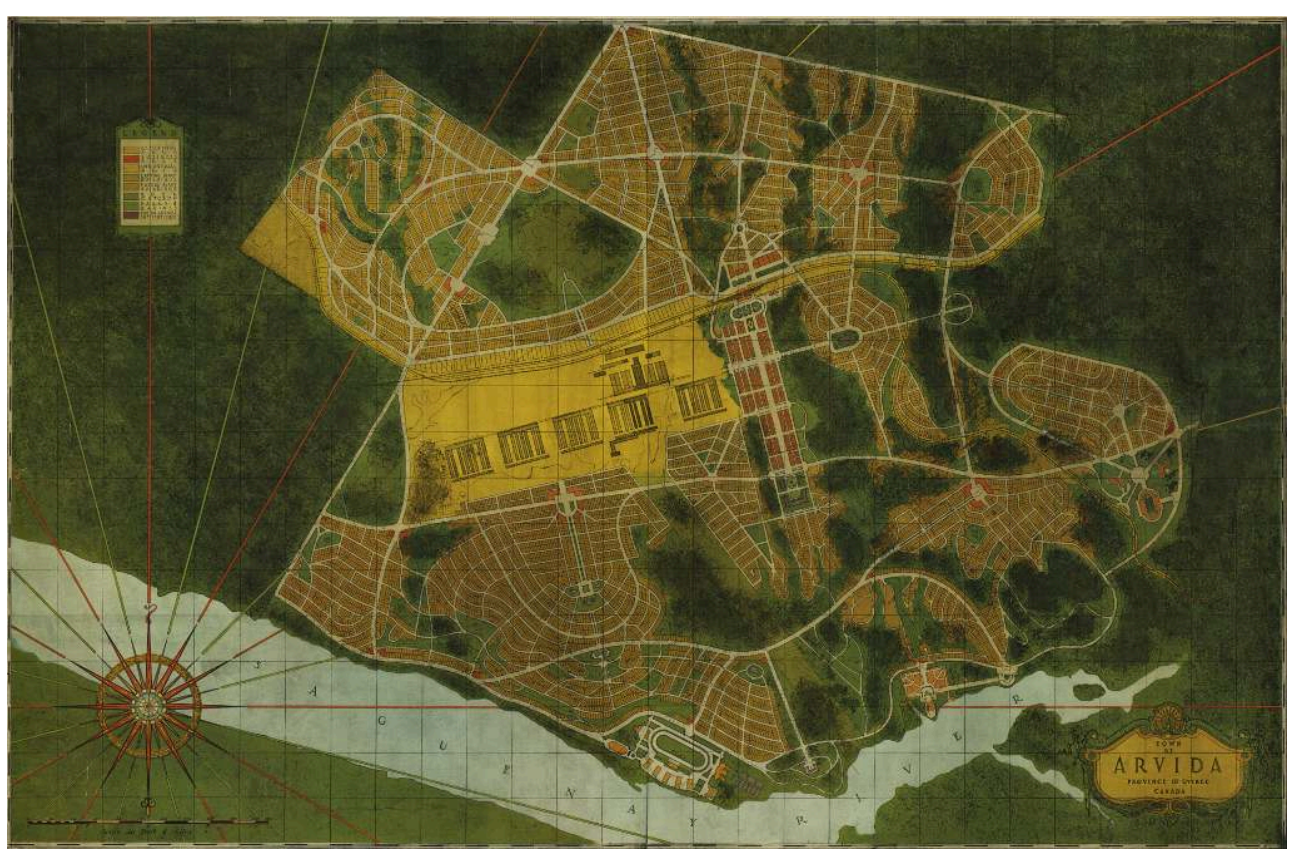

D'autres réalisations récentes présentent un intérêt. I. M. Robinson relève et place l'accélération majeure du phénomène de construction des nouvelles villes extractives nord-américaines dans les années $1950:$ «[...] forty-six new towns came into being in the period 1945 to 1957, each built around a single resource-based industrial enterprise. In the 1950-53 period alone, some eighteen new townsites were created " (Robinson 1962, p. 3). Ces villes méritent notre attention car elles ont une genèse (proximité d'une matière première), une organisation (planification, mono-industrie) et une fin souvent proches ou similaires à celles de nombreuses villes-usines traditionnelles européennes : « many of the earlier townsites died as natural ressources were exhausted or it no longer became economic to exploit them " (ibid., p. 3). Elles ont également le caractère temporaire dont parlait J. D. Porteus pour des company towns plus anciennes : «The company town may be regarded as an essentially temporary pioneering device" (Porteus 1970 p. 129). De plus, toutes ces villes ont été créées ex nihilo sauf Anyox (colonisation d'un territoire du premier peuple Nisga'a) et cela se voit dans leurs toponymes.

32 Ajoutons que toutes ces villes sont isolées et situées dans des marges. Toutes correspondent à des villes pionnières et d'appropriation de territoire, c'est-à-dire à ce qu'ont théorisé J. D. Porteus et L. K. Morisset: villes-frontières, villes pionnières et exploratoires.

Enfin, le paternalisme dans ces villes de compagnie isolées est d'un degré très faible sauf dans quelques cas exceptionnels (Arvida, figure 8). Les équipements ainsi que les bâtiments économiques et sociaux en sont pratiquement absents, les infrastructures de transport vont rarement plus loin. Ce faible degré de paternalisme semble lié à deux éléments principaux : leur isolement en fait des modèles peu visibles, donc à faible valeur symbolique pour des patrons soucieux de leur image; ce sont des villes pensées comme temporaires dans lesquels il n'est pas rentable d'investir autant.

La company town désigne donc, dans ses multiples acceptions, plusieurs types de villesusines nord-américaines ainsi que de nombreuses villes-usines européennes fondées par une seule entreprise. Comme nous l'avons illustré avec le cas d'Arvida et de Chicopee, il existe une certaine porosité entre les modèles européens et les modèles 
nord-américains. Les différences entre les deux modèles sont liées à l'isolement extrême des company towns plutôt qu'à des différences dans la réalisation spatiale du paternalisme: "L'appartenance nationale et philosophique n'est pas, semble-t-il, contrairement à une tendance naturelle à trouver des singularités partout, un facteur pertinent de différenciation en matière d'œuvres paternalistes " (Gueslin 1992 p. 213). En réalité, sur beaucoup de points, les company towns nous aident à comprendre l'essence du modèle européen et à penser des solutions pour les villes-usines postindustrielles.

\section{Apports théoriques et praxéologiques au modèle de la ville-usine : quel potentiel postindustriel ?}

Cette porosité américano-européenne des modèles idéels et concrets des villes-usines et des company towns nous permet d'ouvrir quelques pistes de réflexion inspirées par les company towns sur la ville-usine et sa transition postindustrielle (tableau 1).

Tableau 1 : tableau récapitulatif, apport des modèles nord-américains à la réflexion sur la villeusine (Picon 2019)

\begin{tabular}{|c|c|c|c|c|c|c|}
\hline $\begin{array}{l}\text { Type de } \\
\text { modèle }\end{array}$ & Sous-modèle & $\begin{array}{l}\text { Localisation } \\
\text { principale }\end{array}$ & $\begin{array}{l}\text { Apports } \\
\text { intéressants }\end{array}$ & $\begin{array}{l}\text { Auteurs } \\
\text { principaux }\end{array}$ & exemples & $\begin{array}{l}\text { Notions et pistes } \\
\text { de } \\
\text { redéveloppement } \\
\text { territorial }\end{array}$ \\
\hline $\begin{array}{l}\text { Mill } \\
\text { village }\end{array}$ & / & $\begin{array}{l}\text { Amérique } \\
\text { du Nord }\end{array}$ & $\begin{array}{l}\text { urbanité } \\
\text { relative et } \\
\text { confusion } \\
\begin{array}{ll}\text { avec les } \\
\text { modèles } \\
\text { urbains }\end{array}\end{array}$ & $\begin{array}{l}\text { Garner, } \\
\text { Lorence, } \\
\text { Hirsch et } \\
\text { Hirsch, } \\
\text { Wingerd }\end{array}$ & $\begin{array}{l}\text { Chicopee, } \\
\text { village } \\
\text { modèle }\end{array}$ & $\begin{array}{l}\text { Renforcement de } \\
\text { l'urbanité } \\
\text { centre-ville, } \\
\text { diversité des } \\
\text { fonctions } \\
\text { urbaines }\end{array}$ \\
\hline \multicolumn{7}{|l|}{$\begin{array}{l}\text { Company } \\
\text { town }\end{array}$} \\
\hline & extractive & $\begin{array}{l}\text { Amérique } \\
\text { du Nord }\end{array}$ & $\begin{array}{l}\text { ville- } \\
\text { frontière } \\
\text { (front } \\
\text { pionnier), } \\
\text { ville } \\
\text { temporaire } \\
\text { ou } \\
\text { transitoire, } \\
\text { isolation }\end{array}$ & $\begin{array}{l}\text { Porteus, } \\
\text { Robinson, } \\
\text { Morisset, } \\
\text { Garner }\end{array}$ & $\begin{array}{l}\text { Keno } \\
\text { City, } \\
\text { Uranium } \\
\text { City, } \\
\text { Anyox }\end{array}$ & $\begin{array}{l}\text { Notions de marge } \\
\text { et de marginalité, } \\
\text { villes } \\
\text { rétrécissantes } \\
\text { (Schrumpfung), } \\
\text { soutenabilité } \\
\text { économique }\end{array}$ \\
\hline
\end{tabular}




\begin{tabular}{|l|l|l|l|l|l|l|}
\hline & & & & & & $\begin{array}{l}\text { Hégémonie socio- } \\
\text { spatialer lien } \\
\text { entre } \\
\text { l'attachement } \\
\text { aux héritages } \\
\text { industriel et le } \\
\text { paternalisme, } \\
\text { levier } \\
\text { patrimonial, } \\
\text { paternalisme, } \\
\text { modelage } \\
\text { social } \\
\text { Amériquenabilité } \\
\text { du Nord } \\
\text { sociale } \\
\text { Morisset, } \\
\text { Garner }\end{array}$ \\
\hline
\end{tabular}

Le modèle de la ville-usine de S. Edelblutte (figure 1) est suffisamment ouvert pour subsumer toutes ces nuances de ville-usines, car toutes sont créées ou développées par et pour l'industrie; toutes sont organisées au service de la production; toutes comprennent un habitat ouvrier et, dans les cas les plus paternalistes, des bâtiments économiques et sociaux; toutes comprennent des infrastructures les reliant à l'extérieur, au marché et aux ressources. Les plus petites et les moins sociales sont une version amputée du modèle. Les plus grandes d'entre-elles peuvent être modélisées par un agrégat de diverses versions de ce modèle. À partir des éléments que nous venons d'apporter sur les variations anglophones des modèles de ville-usine, nous allons mener une réflexion sur le devenir postindustriel des territoires ville-usiniers. Cela a un intérêt pratique, car chaque variation du modèle peut avoir des implications sur les difficultés des anciennes villes-usines après leur désindustrialisation, et chacune peut donc avoir une influence sur le redéveloppement territorial d'une ex ville-usine ou d'une ex vallée industrielle constituée d'une coalescence d'anciennes villes-usines (tableau 1).

\section{Caractères transitoire et temporaire ; carences urbaines : le rétrécissement des anciennes villes-usines}

Les villes-usines extractives ont un caractère transitoire ou temporaire comme le montrent les auteurs anglophones au sujet des company towns extractives. Cette dimension n'a pas toujours été anticipée pour les villes-usines européennes. Pour les villes-usines extractives, cela implique un retournement fonctionnel complet au moment où le marché n'est plus rentable ou au moment où la ressource s'épuise. Si le second moment est plutôt facile à anticiper, le premier l'est moins et est à l'origine de nombreuses crises de l'emploi et fermetures de mines. Les villes-usines extractives les plus isolées deviennent alors brutalement des villes rétrécissantes et se résidentialisent ou, pire, deviennent des villes fantômes. Même lorsque leur isolement n'est pas total, elles échappent malgré tout rarement à ces phénomènes (Cwmparc au Pays de Galles par exemple, $c f$. photo 1). De plus, les villes-usines, quel que soit leur type, souffrent d'une carence en urbanité. Les travaux allemands sur la schrumpfung, le rétrécissement brutal des villes d'Allemagne de l'Est, qui considèrent que "[...] l'ampleur $d u$ phénomène invite [...] à envisager la Schrumpfung comme une opportunité pour un changement de paradigme par rapport aux visions classiques des politiques urbaines centrées sur la croissance urbaine» (Florentin et al. 2009, p. 37) sont également une piste de 
réflexion pour un redéveloppement, une transition soutenable et maitrisée de ces villes-usines qui, peut-être, pourrait «valoriser d'autres dimensions telles que la moindre densité, l'urbanité, le patrimoine urbain, la soutenabilité des réseaux, les espaces publics " (ibid., par. 37). «Valoriser l'urbanité » nous renvoie aux problématiques de carence urbaine et signifie qu'il faut redonner une centralité de qualité et une diversité des fonctions urbaines à la ville-usine pour compenser les problématiques de résidentialisation.

Photo 1 (orientation S-N): la ville-usine extractive (charbon) de Cwmparc au Pays de Galles (Picon 2018)

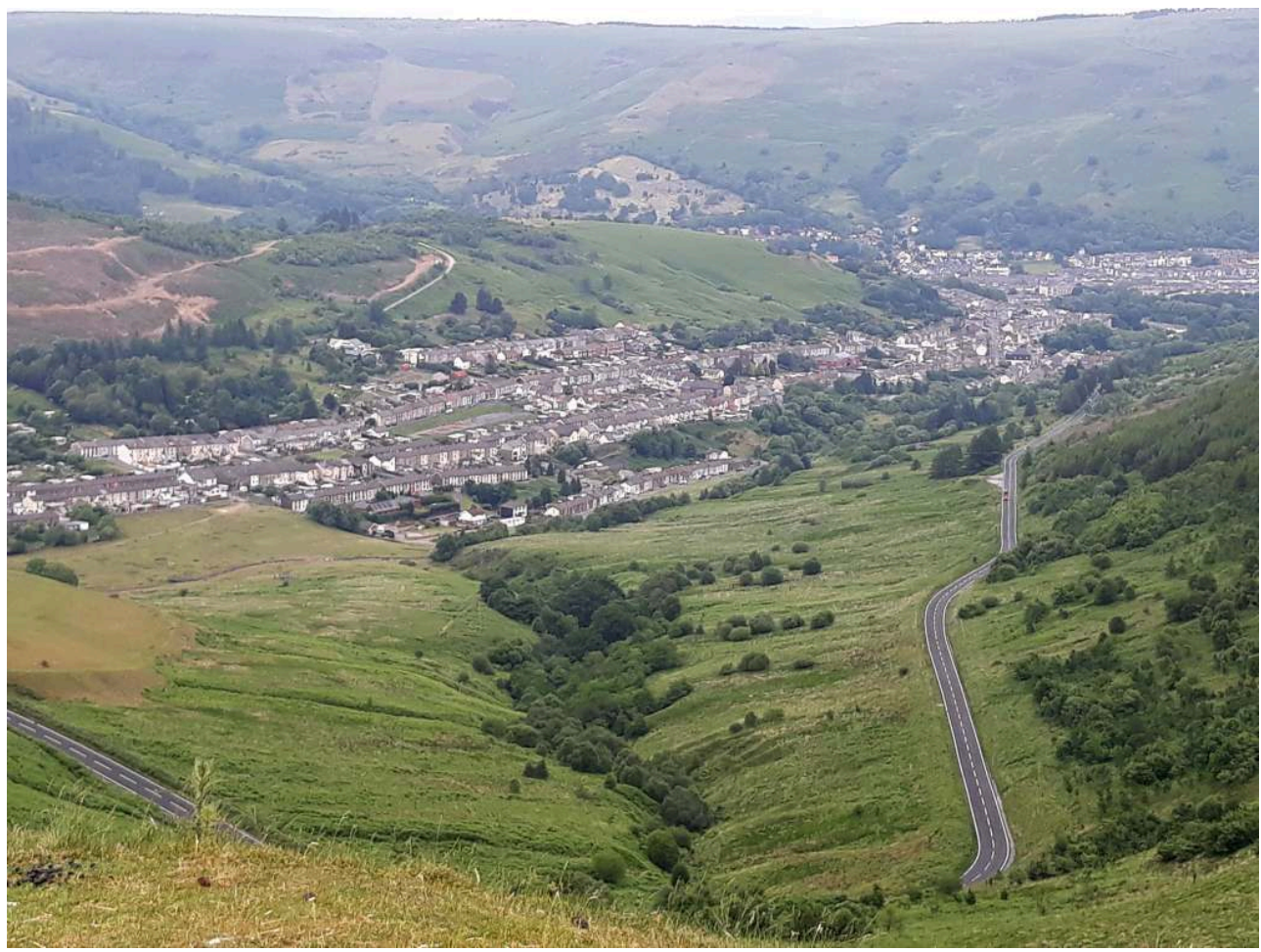

Située en contrebas d'un cirque glaciaire, la ville est isolée, marginalisée dans un cul-de-sac (à l'ouest, plus rien) et ne se développe plus. C'est un long bloc de cités minières qui semble figé dans le temps. Le puits de mine, fermé, était situé à l'extrême ouest de la ville (centre gauche au bord de la photo, au niveau des arbres avant la dernière cité). Les cités sont enchâssées dans une petite vallée affluente de la Rhondda (à droite), une très longue vallée charbonnière saturée d'anciennes cités minières.

Lorsque les villes sont à la fois extractives et manufacturières, elles ont une meilleure résilience car elles peuvent remplacer la ressource locale par une ressource importée souvent plus rentable. De plus, elles sont déjà connectées au marché via leurs infrastructures.

Lorsque les villes sont manufacturières ou mixtes, le caractère temporaire n'est pas systématique, en revanche la transition est nécessaire soit pour que l'industrie survive (transitions vers une industrie plus moderne, haute technicité, marchés de niches) ou que le territoire s'oriente vers d'autres activités économiques (retournement fonctionnel). Cette transition s'accompagne d'une décroissance démographique et urbaine plus ou moins marquée (selon la capacité du territoire à amortir les pertes d'emplois industriels), faisant également de ces ex villes-usines des villes que l'on peut qualifier de rétrécissantes. En général, ce rétrécissement est moins brutal que dans les villes extractives isolées, et si la Schrumpfung est au départ pensée pour les rétrécissements brutaux des villes d'Allemagne de l'Est, c'est aussi un «laboratoire» 
pour des rétrécissements plus lents (ibid., par. 36). Une des solutions que proposent ces travaux sur le rétrécissement urbain est la valorisation du patrimoine urbain, or les villes-usines et company towns disposent encore souvent de leur patrimoine industriel. Pour que la mise en valeur d'un tel patrimoine soit soutenable, il faut notamment que les populations locales y soient attachées. Cet attachement à l'industrie ne provient pas de nulle part, il est souvent lié à la prégnance du paternalisme industriel dans la villeusine et à une nostalgie tardive.

\section{L'hégémonie socio-spatiale des paternalistes : un marqueur spatial toujours prégnant}

Le modelage social décrit par J. D. Porteus fait partie, dans la ville-usine paternaliste, de ce que nous appelons l'hégémonie sociale, au sens gramscien, des patrons dans la villeusine. Cette adaptation du concept s'inspire de la company town de Chicopee (figure 5), où l'accord de la population avec l'ordre social paternaliste était tel qu'il régulait les grèves en douceur (Lorence 2007, p. 302); de celle d'Arvida (figure 7), où l'ordre social était maintenu par la bienveillance et l'égalitarisme : "we can see that the Garnier's "progress in the social order" is translated in the Aluminum Company of America as a certain (and unusual) egalitarianism combined with a benevolent paternalism regarding its workers' needs and futures» (Morisset 2011, p. 30); des premiers mill villages textiles du Sud des Etats-Unis (figure 1), terreaux d'un paternalisme beaucoup plus dur; de la ville-usine de Jœuf dans le Pays-Haut Lorrain (figure 6), dont l'espace est très marqué par les idées du catholicisme social (cf. I.C.).

41 L'hégémonie sociale a une influence considérable sur l'espace ville-usinier, nous allons donc la détailler. Dans la partie de ses carnets rédigée entre 1930 et 1932, A. Gramsci définit l'hégémonie sociale que certaines superstructures peuvent avoir sur une population. Le concept a souvent été repris et adapté par les sciences sociales (en Géographie, voir notamment Sevilla-Buitrago, 2017). A. Gramsci considère qu'il faut deux critères pour exercer une hégémonie sur un groupe social à l'échelle d'un pays ou d'une "superstructure »: "1. de l'accord "spontané» donné par les grandes masses de la population à l'orientation imprimée à la vie sociale par le groupe fondamental dominant [...] ;

2. De l'appareil de coercition [...]; mais cet appareil est constitué pour l'ensemble de société en prévision des moments de crise [...], lorsque l'accord spontané vient à faire défaut » (Gramsci 2014, p. 230)

Pour que cela fonctionne à l'échelle de la ville-usine, ces deux critères doivent s'appliquer dans un pays compatible avec le paternalisme industriel « d'entreprise ». Ils n'auraient pas fonctionné, en tout cas à cette échelle, dans l'Union des Républiques Socialistes Soviétiques (URSS). L'accord spontané est bien plus important dans les systèmes les plus philanthropiques, plus en retrait dans les systèmes plus paternalistes. Le meilleur moyen d'obtenir un accord « spontané » est tout simplement de satisfaire la population ouvrière, en ce sens, c'est un aspect plutôt positif du paternalisme. L'autre aspect de cet accord, c'est le contrôle et la surveillance plus ou moins tacites de la population, un aspect plutôt négatif du paternalisme, qu'il soit philanthropique ou non. Cependant, c'est le critère le plus essentiel des deux pour que l'hégémonie sociale soit assurée dans la ville-usine, le second critère n'étant qu'une goupille de sécurité comme le dit A. Gramsci. Cette hégémonie sociale construit, place, organise, assigne et marque 
l'espace de la ville-usine d'une manière particulière. C'est pour cela que nous parlerons désormais d'hégémonie socio-spatiale.

Bien entendu, cette hégémonie n'est jamais parfaite et ne s'exerce jamais absolument, son équilibre tient à la tension entre l'accord spontané de la population et son désaccord (grèves, révoltes souvent liées à des licenciements ou aux conditions de travail). Lorsque la balance penche trop du côté du désaccord, que des grèves ou des révoltes éclatent, soit on réprime, soit on négocie pour renforcer l'accord spontané. Ces deux critères ont été exprimés autrement par de nombreux auteurs anglophones et francophones. Il peut sembler peu utile de les formaliser, mais nous le faisons car cela a un intérêt praxéologique pour l'aménageur qui doit penser le redéveloppement territorial.

En effet, concevoir ainsi le contrôle et la bienveillance des patrons paternalistes permet de comprendre comment est organisé l'espace et pourquoi, et dans quels types de villes-usines l'attachement aux héritages de l'industrie paternaliste est le plus fort: nous posons l'hypothèse que plus l'accord "spontané ", garant de l'ordre social paternaliste, a été entretenu, plus cet attachement sera fort. L'enquête que nous avions menée en 2016 sur les villes-usines paternalistes des vallées sidérurgiques de l'Orne et de la Fensch dans le territoire lorrain allait dans ce sens puisque en 2016 « $63 \%$ de l'effectif souhaite une conservation du patrimoine industriel» (Picon 2019, p. 50) dans ces vallées. Ce phénomène était encore plus marqué chez ceux qui avaient connu le paternalisme à son apogée: « $68 \%$ des plus de cinquante ans souhaitent une conservation du patrimoine industriel, alors que ce n'est seulement le cas que de 57\% des moins de cinquante ans » (ibid., p. 50). Cependant, il est important de nuancer ces résultats. Le désir de conservation du patrimoine industriel apparaît ici cinquante ans après le début de la crise de l'industrie locale. Avant d'en arriver là, le territoire traversé par le déclin industriel passe souvent par une phase d'incrédulité durant laquelle il ne se passe rien et une phase de deuil qui mène à des destructions irréfléchies pour effacer toutes les traces douloureuses de l'industrie (Grossetti et al. 1998 ; Edelblutte 2012 p. 99). Si les héritages industriels ne survivent pas au deuil, dont la violence est proportionnelle aussi à la prégnance de l'industrie dans la vie de la population (et donc à l'intensité du paternalisme), il est trop tard pour utiliser ce patrimoine pour redévelopper ces territoires. La survie de ces héritages malgré la phase de deuil est souvent due à des fermetures tardives ou à la paralysie du foncier par les entreprises qui évitent ainsi de dépolluer et de payer (mise sous cocon des hauts-fourneaux de Hayange par ArcelorMittal par exemple). C'est notamment ce qui a fait la différence entre la vallée de l'Orne et de la Fensch dans le Pays Haut lorrain (Picon 2019).

\section{La ville-usine paternaliste planifiée : un potentiel pour soigner la qualité urbaine de la ville contemporaine}

Enfin, il faut évoquer les trois critères de L. K. Morisset qui permettent à la ville-usine de tendre vers l'utopie la plus philanthropique et le cadre de vie le plus agréable: l'idéalisme des patrons, leur expérience en matière d'aménagement, un cadre géographique exceptionnel. Ces trois derniers critères participent triplement à la possibilité d'une patrimonialisation de la ville-usine. D'abord, ils permettent l'accord « spontané » des populations avec l'ordre social paternaliste de leur ville (et donc plus 
tard à une volonté de conserver les héritages industriels). Ensuite, ils donnent des caractères uniques et attachants à l'architecture et à l'urbanisme d'une ville, favorisant ainsi sa patrimonialisation. En somme, lorsque les patrons ont «soigné le rapport affectif " à la ville-usine, ils ont permis aux aménageurs d'aujourd'hui de «soigner le rapport affectif » à ce que la ville est devenue: «monuments culturels, patrimoniaux, ambiance et animation des espaces publics, activités de loisirs, etc. sont autant d'éléments construisant le rapport affectif des citoyens à leur ville. [...] La prise en compte du rapport affectif doit évoluer en même temps que les besoins des citadins et nous conforte dans l'idée que la ville doit toujours être repensée et vivante, comme un organisme répondant aux besoins d'une population et à une demande sociale» (Del Biondo 2014, p. 119). La difficulté avec ce genre de pratique, c'est de ne pas gentrifier la ville, c'est-à-dire de respecter son identité sociale. Pour cela, il faut considérer la ville et le patrimoine comme vivants, sans cesse en mutation, éviter la muséification et tenir compte des besoins des habitants comme le préconise L. Del Biondo.

\section{Conclusion}

Les company towns nord-américaines et les villes-usines européennes peuvent sembler bien différentes au premier abord, en mener une comparaison stricto sensus est évidemment impossible. En revanche, la porosité entre les deux modèles permet, en étudiant les unes, de repenser les autres et d'en extraire l'essence commune (parties I et II). Celle-ci a un intérêt pratique car les problématiques des ex villes-usines mises en exergue par cette synthèse révèlent à la fois leurs fragilités héritées et leur potentiel postindustriel. Ceux-ci dépendent de nombreux facteurs dont nous avons proposé un aperçu : marginalité, isolement, planification et hiérarchisation de l'espace, degré de paternalisme, etc. (partie III). Pour assurer leur transition maîtrisée vers des villes de qualité, et donc pour assurer le redéveloppement territorial des territoires qu'elles forment, il faut à la fois s'appuyer sur leur potentiel postindustriel particulier (patrimoine, situation, desserte, industrie encore active, etc.) et mener une réflexion sur leurs difficultés héritées: marginalité, carences urbaines, éclatement urbain, coupures urbaines, dépendance mono-industrielle, saturation de l'espace, etc. Pour cela, la connaissance fine de leur morphogenèse urbaine est indispensable : degré de paternalisme industriel, contexte de création, planification antérieure, etc. Si chaque ville-usine est unique, s'il faut une solution particulière à chaque territoire, la détermination de caractéristiques communes à chaque nuance de ce modèle permettra, peut-être, d'en déduire un potentiel postindustriel typique et des grands principes de redéveloppement territorial applicables à chaque variation du modèle. Afin de perfectionner ces principes, il faudrait ajouter à cette synthèse les villes-usines sudaméricaines qui ont également une origine européenne (à la genèse proto-industrielle coloniale et souvent agro-industrielle : voir Dorel-Ferré - dir. 2016, p. 168-223) et, de manière générale, l'ensemble des villes-usines du monde qui fut influencé et/ou colonisé par les puissances d'Europe de l'Ouest, notamment celles du continent africain, peu étudiées, mais aussi celles de l'Inde britannique, plus connues. Enfin, il a existé d'autres villes-usines plus à part, qu'il serait aussi judicieux d'intégrer à une telle synthèse, ne serait-ce que pour analyser ce qui les sépare de leurs cousines, mais aussi pour en extraire l'essence commune. Ces villes-usines, plus «exotiques», se retrouvaient notamment en URSS pendant la Guerre Froide (villes-usine extractives monumentales en Sibérie telle que Mirny, villes-usines secrètes dédiées à l'armement 
nucléaire), plus précocement en Scandinavie avec les fameux Bruk suédois protosidérurgiques, ou en Chine (où se mêlent dans la première moitié du XXe siècle des influences occidentales, soviétiques et japonaises).

\section{BIBLIOGRAPHIE}

Bailly A. S., Aydalot P., Godbout J., Hussy C., Raffestin C., Turco A., 1983, « La marginalité : réflexions conceptuelles et perspectives en géographie, sociologie et économie », Géotopiques, p. 73-115.

Blésius J.-C. (2014), « Vivre avec les industries ? La prise en compte du risque industriel majeur dans les pratiques d'aménagement. Une approche comparée entre Québec et France ", Territoire en mouvement Revue de géographie et aménagement, $\mathrm{n}^{\circ}$ 23-24, p. 115-128

Bruyelle, P. Dezert B., 1983, « Les relations entre la ville et l'industrie : formes anciennes et formes nouvelles ", Hommes et Terres du Nord, vol. 1, $n^{\circ}$ 1, p. 7-12

Byington M. F., 1909, « The Family in a Typical Mill Town », American Journal of Sociology, vol. 14, $n^{\circ} 5$, p. 648-659

Capdevielle, J. \& Mouriaux R., 1975, L'ouvrier conservateur, Les cahiers du laboratoire d'études et de recherches sociologiques sur la classe ouvrière, $102 \mathrm{p}$.

Colocousis C. R., 2012, « “It Was Tourism Repellent, That's What We Were Spraying”: Natural Amenities, Environmental Stigma, and Redevelopment in a Postindustrial Mill Town », Sociological Forum, vol. 27, $\mathrm{n}^{\circ}$ 3, p. 756-776

Crawford M., 1995, Building the workingman's paradise: the design of American company towns, Verso, Londres, $256 \mathrm{p}$.

Daviet S., 2006, «L'évolution du concept de reconversion : de la substitution d'activité au redéveloppement des territoires » in Daumalin X \& Mioche P. (dir.), Territoires européens du charbon : Des origines aux reconversions, p. 243-255

Del Biondo L., 2014, Les stratégies de recomposition urbaine soutenable des anciens territoires industrialo-urbains. Université de Lorraine, Nancy, 427 p.

Del Biondo L. \& Edelblutte S., 2016, « Le paysage des anciennes villes-usines européennes : un nouveau patrimoine entre négation, alibi, reconnaissance et complexité des jeux d'acteurs », Annales de géographie, $\mathrm{n}^{\circ}$ 711, p. 466-489

Dinius O. J. (dir.), Vergara A. (dir.), Eakin M. C., Esch E., Feliú E., Gómez-Galvariato A., Herod A., 2011, Company Towns in the Americas: Landscape, Power, and Working-Class Communities, University of Georgia Press, Athènes, 236 p.

Dorel-Ferré G. (dir.), 2016, Villages ouvriers et villes-usines à travers le monde, Chambéry, Université de Savoie Mont Blanc, $288 \mathrm{p}$.

Dorel-Ferré G. (dir.) ,2019, Le patrimoine industriel dans tous ses états : hommage à Louis Bergeron. Chambéry, Université de Savoie, $463 \mathrm{p}$. 
Dorel-Ferré G. \& Bergeron L., 1996, Le patrimoine industriel : un nouveau territoire, Paris, Liris, 127 p. Doyen J.-P., 1983, « Les villes-usines de la moyenne Moselle », Annales de La Société d'émulation Des Vosges, p. 52-71

Edelblutte S., 2009, Paysages et territoires de l'industrie en Europe, Paris, Ellipses, 272 p.

Edelblutte S., 2010, «Paternalisme et territoires politiques dans la France de la seconde révolution industrielle : Un regard rétrospectif sur les liens entre firmes et territoires communaux », Revue Géographique de l'Est, vol. 50, n 3-4, p. 1-15

Edelblutte S., 2012, Paysages industriels en Europe : constructions, reconversions, patrimonialisations. Université de Lorraine, Nancy, $186 \mathrm{p}$.

Edelblutte S., 2014, « Reconversion industrielle ou redéveloppement territorial ? L'exemple de Thaon-les-Vosges, ancienne ville-usine textile lorraine », Géoconfluences, p. 1-6

Edelblutte S. \& Legrand J., 2012, « Patrimoine et culture industriels en milieu rural : quelles spécificités? ", Revue Géographique de l'Est, vol. 52, n 3-4

Engels F., 1969, La question du logement (1872), Les éditions sociales, Paris, 110 p.

Férérol M.-E., 2014, « Les petites villes des espaces interstitiels et l'industrie : un couple indissociable ? L'exemple du sud Massif Central », Territoire en mouvement, Revue de géographie et aménagement, $\mathrm{n}^{\circ} 23-24, \mathrm{p} .15-27$

Florentin D., Fol S. \& Roth H., 2009, « La “Stadtschrumpfung” ou "rétrécissement urbain” en Allemagne : un champ de recherche émergent », Cybergeo : European Journal of Geography Foucault M., 2008, Surveiller et punir : naissance de la prison, Paris, Gallimard, 360 p.

Freitag B., 2005, « Le familistère de guise un projet utopique réussi », Diogène, $\mathrm{n}^{\circ}$ 209, p. 101-108

Garner J. S. (dir.), 1984, The Model Company Town : Urban Design Through Private Enterprise in Nineteenth-Century New England, University of Massachusetts Press, 312 p.

Garner J. (dir.), 1992, The Company Town: Architecture and Society in the Early Industrial Age, New York, Oxford University Press, $256 \mathrm{p}$.

Garner J. S., 1971, « Leclaire Illinois : A Model Company Town (1890-1934) », Journal of the Society of Architectural Historians, vol. 30, $\mathrm{n}^{\circ}$ 3, p. 219-227

Gramsci, A. (2014). Textes choisis. Pantin, Le Temps des cerises, 338 p.

Grossetti M., Beslay C., Salles D., Guillaume R., Daynac M. \& Tautelle F., 1998, La construction des politiques locales. Reconversions industrielles et systèmes locaux d'action publique, Paris, L'Harmattan, $224 \mathrm{p}$.

Gueslin A., 1992, « Le paternalisme revisité en Europe occidentale (seconde moitié du XIXe siècle, début du XXe siècle) ", Genèses. Sciences sociales et histoire, vol. 7, nº 1, p. 201-211

Jalabert G \& Grégoris M., 1987, Turin : de la ville-usine à la technopole. Annales de Géographie, $\mathrm{n}^{\circ}$ 538 , p. 680-704

Hirsch J. \& Hirsch K., 2002, « Disability in the Family?: New Questions about the Southern Mill Village », Journal of Social History, vol. 35, nº 4, p. 919-933

Hodges N. \& Frank P., 2014, « Reinventing “Towel City, Use”: textiles, tourism, and the future of the Southeastern mill town », Family and Consumer Sciences Research Journal, nº 2, p.173-187

Le Play F., 1870, L'organisation du travail, Paris, Economica : Anthropos, 360 p. 
Lorence J. J., 2007, « The Workers of Chicopee: Progressive Paternalism and the Culture of Accommodation in a Modern Mill Village ", The Georgia Historical Quarterly, vol. 91, n 3, p. 292-323

Luxembourg C., 2008, Villes en transition : les avatars de l'industrie dans les villes françaises petites et moyennes (Blagnac, Bourges, Gennevilliers, Le Creusot, Valenciennes), Paris, Université de Nanterre Paris X, $337 \mathrm{p}$.

Luxembourg C., 2014, Métamorphoses des villes industrielles : vivre la ville désindustrialisée, Paris, Harmattan, $176 \mathrm{p}$.

Mathis D. \& Mathis A., 2017, « Régénération de la conurbation sidérurgique du Val-de-Fensch (Moselle-France) », Territoire en mouvement, Revue de géographie et aménagement

Minnery J., 2012, « Model industrial settlements and their continuing governance », Planning Perspectives, vol. 27, $n^{\circ}$ 2, p. 309-321

Morisset L. K., 2011, « Non-Fiction Utopia : Arvida,Cité Industrielle Made Real », Journal for the Study of Architecture in Canada, vol. 36, $\mathrm{n}^{\circ} 1$

Morisset L. K., 2017, « Les « villes de compagnie » du Canada. Un patrimoine urbain pour le vivre ensemble de notre siècle? », Entreprises et histoire, $\mathrm{n}^{\circ} 87$, p. 39-50

Mosher A. E., 1995, « “Something Better Than the Best”: Industrial Restructuring, George McMurtry and the Creation of the Model Industrial Town of Vandergrift, Pennsylvania, 1883-1901 », Annals of the Association of American Geographers, vol. 85, nº 1, p. 84-107

Mullin J., 1998, « Mill town roots », Planning, vol. 64, n 3

Murard L. \& Zylberman P., 1977, « Le petit travailleur infatigable ou le prolétaire régénéré, Villesusines, habitat et intimités au XIXe siècle », Recherches, $n^{\circ} 176,217$ p.

Nacé C. \& Nacé J.-R., 2008, « L'Ardèche, terre de patrimoine industriel ? », Historiens et Géographes, $\mathrm{n}^{\circ} 401$, p. $277-284$

Picon M., 2019, « L'Orne et la Fensch : deux anciennes vallées industrielles lorraines, deux redéveloppement territoriaux ", En détail. Regards croisés sur les territoires industriels, Paris, CGET, p. $43-50$

Pollard S., 1964, « The Factory Village in the Industrial Revolution », The English Historical Review, $\mathrm{n}^{\circ} 312,513-531$

Porteous J. D., 1970, « The Nature of the Company Town », Transactions of the Institute of British Geographers, $n^{\circ}$ 51, p. 127-142

Reclus E., 1905, L'Homme et la Terre, Paris, Librairie universelle, 594 p.

Robinson I. M., 1962, New industrial towns on Canada's resource frontier, Chicago, University of Chicago, $190 \mathrm{p}$.

Sevilla-Buitrago A., 2017, « Gramsci and Foucault in Central Park: Environmental hegemonies, pedagogical spaces and integral state formations ", Environment and Planning D: Society and Space, vol. $35, n^{\circ} 1$, p. $165-183$

Solecki W. D., 1996, « Paternalism, pollution and protest in a company town », Political Geography, vol. $15, n^{\circ} 1$, p. $5-20$

Wingerd M. L., 1996, « Rethinking Paternalism: Power and Parochialism in a Southern Mill Village », The Journal of American History, vol. 83, n 3, p. 872-902 
Wirth P., Cernic-Mali B., \& Fischer W., 2012, Post-Mining Regions in Central Europe : Problems, Potentials, Possibilities, Munich, Oekom Verlag Gmbh, 269 p.

\section{NOTES}

1. La manufacture royale d'Arc-et-Senans, située à Arc-et-Senans dans le Doubs, étaient une utopie (concrétisée) proto-industrielle précoce dédiée à l'exploitation du sel gemme. Il s'agit désormais d'un des plus célèbres patrimoines proto-industriels français et le site est inscrit à la liste du patrimoine mondial de l'UNESCO.

2. Ces deux villes visaient respectivement à découvrir des parties inconnues du bassin houiller de la Sarre et du bassin ferrifère du Pays Haut. Elles se situaient dans les marges du territoire français au moment de leur métamorphose en ville-usine (frontière franco-allemande).

3. Les concepteurs de la ville ont tenu compte de sa topographie très ravinée et ont ajouté des espaces verts dans les ravines afin de les intégrer soigneusement au tissu urbain, un exemple majeur de « nature en ville».

\section{RÉSUMÉS}

La grande variété des villes-usines en Europe de l'ouest et des company towns en Amérique du Nord ne permet pas de mener une comparaison stricte de ces territoires. En revanche, la porosité entre les différents modèles permet d'en extraire des caractéristiques communes. Au travers d'une synthèse mêlant étude bibliographique et terrain, nous pouvons deviner quelques-unes de ces caractéristiques et en déduire, pour chacune d'entre-elle, les faiblesses et le potentiel postindustriel dont peuvent hériter ces villes-usines. Ceux-ci sont un premier matériel de réflexion pour déterminer des grands principes de redéveloppement territorial applicables à ces anciens territoires industrialo-urbains.

The diversity of factory towns (or mill towns) in Western Europe and the diversity of company towns in North America do not allow us to compare these territories. However, the permeability between these models allows us to identify their common features. We should be able to guess some of these features by synthetising articles, books and field research. Finally, these features allow us to identify the weaknesses and the postindustrial potential of the european mill towns. These results are first potential solutions to regenerate theses old industrial and urban territories. 


\section{INDEX}

Keywords : ville-usine, ville de compagnie, ville industrielle, vallée industrielle, company town, village-usine, mill village, factory town, mill town, Western Europe, North America, urban regeneration, transition, fringe, shrinking cities, paternalism, urban landscape, industrial heritage

Mots-clés : ville-usine, ville de compagnie, ville industrielle, vallée industrielle, company town, village-usine, mill village, factory town, mill town, Europe de l'ouest, Amérique du Nord, redéveloppement territorial, transition, marges, villes rétrécissantes, paternalisme, urbanité, patrimoine industriel

\section{AUTEUR}

\section{MICHAËL PICON}

Doctorant et ATER en géographie - LOTERR, centre de recherche en géographie - Université de Lorraine - Campus Lettres et Sciences Humaines - 23, boulevard Albert Ier - 54000 NANCY michael.picon@univ-lorraine.fr 